Monatsschr Kinderheilkd 2010 • 158:214-215

DOI 10.1007/s00112-009-2138-z

Online publiziert: 17. Februar 2010

(c) Springer-Verlag 2010
P. Bader ${ }^{1}$. D. Reinhardt ${ }^{2}$

${ }^{1}$ Schwerpunkt Stammzelltransplantation, Klinik für Kinder- und Jugendmedizin III, Frankfurt am Main

${ }^{2}$ Kinderklinik und Poliklinik, Dr. von Haunersches Kinderspital, München

\title{
Pädiatrische Stammzelltherapie
}

Ende der 196oer Jahre wurde von der Gruppe um R.A. Good in Minneapolis die erste erfolgreiche allogene Transplantation bei einem Kind mit einem schweren kombinierten Immundefekt durchgeführt. Diese begründete die „moderne Ära"der Stammzelltransplantation (SZT). Ein Jahr später wurde in Seattle erstmals einem Leukämiepatienten das Knochenmark eines HLA-identischen (HLA: „,human leukocyte antigene") Geschwisters übertragen. Seither hat sich die allogene SZT zu einem sehr wertvollen Therapieverfahren in der Behandlung vieler Patienten mit Hochrisikoleukämien, aber auch für Patienten mit nichtmalignen Erkrankungen der Hämatopoese und des Immunsystem sowie mit Stoffwechselstörungen entwickelt.

\section{( Die möglichen Langzeitfolgen der SZT müssen weiter verringert werden}

Trotz großer Fortschritte in der Transplantationsmedizin bleibt die allogene SZT eine einschneidende Therapieform mit vielen akuten Komplikationsmöglichkeiten sowie der Gefahr von Langzeitfolgen wie Wachstums- und Fertilitätsstörungen. Diese Komplikationen sind zumeist der hochdosierten Chemo-/Radiotherapie geschuldet; aber auch immunologische Komplikationen wie die T-Zell-vermittelte „Transplantat versus Empfängererkrankung“ („graft versus host disease", GvHD) sind geeignet, langfristige Beeinträchtigungen zu hinterlassen. Gerade in der Kinder- und Jugendmedizin sind Langzeitfolgen von besonderer Bedeutung; es sollten alle Anstren- gungen unternommen werden, Langzeitfolgen zu verhindern, die die überlebenden Kinder stigmatisieren und eine altersgerechte Entwicklung unmöglich machen.

\section{(7) Die immunologische Wirkung des Transplantats hat entscheidende Bedeutung}

Da nur etwa $25 \%$ aller Patienten über einen HLA-identischen Spender in der Familie und weitere $50 \%$ über einen passenden unverwandten Spender verfügen, ist es ein zentrales Ziel, den Spenderpool zu erweitern und über HLA-Barrieren hinweg Stammzellen in Form von Knochenmark, peripherem Blut und Nabelschnurblut zu transplantieren. Vor allem im pädiatrischen Bereich stellt dabei der Einsatz von haploidentischen Spendern (im Wesentlichen Eltern) zum Stammzellersatz nach intensiver Radio-/Chemotherapie einen wesentlichen Fortschritt dar.

Lange Zeit wurde zur erfolgreichen Behandlung von Patienten mit bösartigen Erkrankungen eine hochintensive, möglichst „supraletale“ Chemo-/Radiotherapie für erforderlich gehalten, wobei der Stammzellersatz im Kurationskonzept eine nachgeordnete Rolle spielte. Die intensive Beschäftigung mit den immunologischen Auswirkungen der Übertragung großer Mengen von Stammzellen und insbesondere der Subfraktionen des Transplantats zeigte jedoch die Bedeutung der immunologischen Wirkungsweise des Transplantats selbst, was zu einem Paradigmenwechsel führte. So spielen NK-Zellen im Transplantat bei der Überwindung von malignen Erkrankungen eine entschei- 
dendere Rolle als die der Transplantation vorausgehende Chemo-/Radiotherapie. Damit können allogene Transplantationsverfahren und insbesondere die haploidentische Transplantation als immunologische Plattform verstanden werden, die eine sorgfältige Auswahl und Präparation des Transplantats selbst und eine vielfältige Kombination mit zellulären Therapien nach der Transplantation erlaubt. Die hochdosierten Chemo-/Radiotherapien sind weniger für die Eradikation des malignen Zellklons notwendig, sondern werden vielmehr als Ursache für viele unerwünschte Effekte der Transplantationsverfahren verstanden: Bei Kindern sind dies die negativen Auswirkungen auf Wachstum und Entwicklung und die Gewebeschädigung, die die Kaskade hin zur akuten und chronischen Graft-versus-HostReaktion in Gang setzt.

Dies eröffnet eine Perspektive, die allogene Transplantationsverfahren in $\mathrm{Zu}$ kunft mit reduzierten Konditionierungen ohne wesentliche Gewebeschädigung oder sogar mit ausschließlich immunologischen Vorbereitungen des Empfängers denkbar macht. Spezifische Zelltherapieelemente sollen dabei zielgerichtet gegen die zugrunde liegende maligne Erkrankung eingesetzt werden, ohne eine allogene GvHD auszulösen. Andererseits können im Rahmen der SZT spezifische T-Zellen auch gegen Viren und Pilze verabreicht werden; beides sind Maßnahmen, die die allogene SZT weiter sicherer und erfolgreicher machen werden.

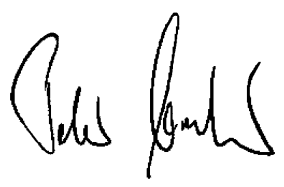

Prof. Dr. P. Bader

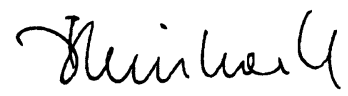

Prof. Dr. D. Reinhardt

\section{Korrespondenzadresse \\ Prof. Dr. P. Bader}

Schwerpunkt Stammzelltransplantation, Klinik für Kinder- und Jugendmedizin III, Frankfurt am Main, Theodor-Stern-Kai 7, 60590 Frankfurt am Main, peter.bader@kgu.de
„Monatsschrift Kinderheilkunde“ bietet Ihnen jeden Monat umfassende und aktuelle Beiträge zu interessanten Themenschwerpunkten aus allen Bereichen der Pädiatrie.

Wir haben die Jahrgänge 2009/2010 im Überblick für Sie zusammengestellt:

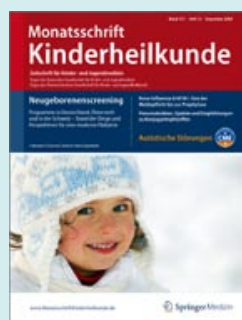

2009

- Heft 01/09 Entscheidungen am Lebensende in der Pädiatrie

- Heft 02/09 Das Kind mit schwerer chronischer Erkrankung

- Heft 03/09 Nephrotisches Syndrom

- Heft 04/09 Spätfolgen in der pädiatrischen Onkologie

- Heft 05/09 Kinder \& Medien

- Heft 06/09 Das Schreikind

- Heft 07/09 Knochenschmerzen

- Heft 08/09 Neue Impfungen

- Heft 09/09 Angeborene Immundefekte

- Heft 10/09 Frühförderung

- Heft 11/09 Zerebralparesen/Neuropädiatrie

- Heft 12/09 Neugeborenenscreening

2010

- Heft 01/10 Neue Morbiditäten in der Pädiatrie

- Heft 02/10 Asthma bronchiale

- Heft 03/10 Pädiatrische Stammzelltherapie

- Heft 04/10 Politische Kindermedizin

- Heft 05/10 Motorische Entwicklungen

- Heft 06/10 Prävention

- Heft 07/10 Neuroophthalmologie

- Heft 08/10 Chronisch-entzündliche Darmerkrankungen

- Heft 09/10 Depressive Störungen im Kindesalter

- Heft 10/10 Kinderkardiologie

- Heft 11/10 Hepatitis

- Heft 12/10 Der auffällige Urinbefund

\section{Beiträge auf einen Blick}

Abonnenten haben online Zugriff auf alle Beiträge im elektronischen Volltextarchiv unter

\section{www.MonatsschriftKinderheilkunde.de}

\author{
Ins Volltextarchiv gelangen Sie von der Startseite über den Navigationspunkt \\ "Online-Archiv".
}

Eine interessante Lektüre wünscht Ihnen

Ihre Redaktion

Fachzeitschriften Medizin/Psychologie 